\title{
B-type Natriuretic Peptide During Treatment with Sacubitril/Valsartan: the PARADIGM-HF Trial
}

\author{
Brief Title: BNP in PARADIGM-HF
}

Peder Langeland Myhre, MD PhD ${ }^{1,2^{*}}$; Muthiah Vaduganathan, MD MPH ${ }^{1^{*}}$; Brian Claggett, $\mathrm{PhD}^{1}$; Milton Packer, MD ${ }^{3}$; Akshay S. Desai, MD MPH ${ }^{1}$; Jean L. Rouleau, MD'; Michael R. Zile, MD Karl Swedberg, $\mathrm{MD}^{6}$; Martin Lefkowitz $\mathrm{MD}^{7}$; Victor Shi, MD ${ }^{7}$; John J.V. McMurray, MD ${ }^{8}$; Scott D. Solomon $\mathrm{MD}^{1}$

${ }^{1}$ Cardiovascular Division, Brigham and Women's Hospital, Boston, MA, USA

${ }^{2}$ Division of Medicine, Akershus University Hospital and University of Oslo, Norway

${ }^{3}$ Baylor University Medical Center, Dallas TX, USA

${ }^{4}$ Montreal Heart Institute and University of Montreal, Montreal, QC, Canada

${ }^{5}$ Medical University of South Carolina and Ralph H. Johnson Veterans Administration Medical Center, Charleston, SC, USA

${ }^{6}$ University of Gothenburg, Gothenburg, Sweden

${ }^{7}$ Novartis Pharmaceuticals Corporation, East Hanover, NJ, USA

${ }^{8}$ University of Glasgow, Glasgow, UK

* Both authors contributed equally as co-first authors

\section{FUNDING}

The PARADIGM-HF trial was funded by Novartis AG.

\section{DISCLOSURES}

Dr. Myhre has received speaker fees from Novartis and is supported by a postdoctoral research grant from the South-Eastern Norway Regional Health Authority (Dr. Røsjø), Akershus University Hospital, Norway (Dr. Omland), the Norwegian Medical Association and the Unger Vetlesen Medical Fund. Dr. Vaduganathan is supported by the KL2/Catalyst Medical Research Investigator Training award from Harvard Catalyst | The Harvard Clinical and Translational Science Center (NIH/NCATS Award UL 1TR002541), and serves on advisory boards for AstraZeneca, Bayer AG, and Baxter Healthcare. Dr. Packer has consulted for Amgen, Actavis, AstraZeneca, Bayer, Boehringer Ingelheim, Daiichi Sankyo, Pfizer, Relypsa, Sanofi, Cytokinetics, and Cardiorentis. Prof McMurray's employer, University of Glasgow, was paid by Novartis for Prof McMurray's time spent as co-chairman of the PARADIGM-HF trial. Dr. Desai has received research grant support from Novartis and consulting fees from Novartis, AstraZeneca, Abbott, Relypsa, and DalCor Pharma. Dr. Swedberg has received honoraria from Novartis for sponsored lectures. Dr. Zile is a consultant for and has received grants from Novartis. Dr. Solomon has received research grants from Alnylam, Amgen, AstraZeneca, Bellerophon, Celladon, Gilead, GlaxoSmithKline, Ionis Pharmaceutics, Lone Star Heart, Mesoblast, MyoKardia, NIH/NHLBI, Novartis, Sanofi Pasteur, Theracos, and has consulted for Alnylam, Amgen, AstraZeneca, Bayer, Bristol-Myers Squibb, Corvia, Gilead, GlaxoSmithKline, Ironwood, Merck, Novartis, Pfizer, Takeda, and Theracos. All other authors report no other relationships relevant to the contents of this paper to disclose.

\section{ACKNOWLEDGEMENTS}

None

Word Count: 4,135

Address for Correspondence: Dr. Scott D. Solomon, Cardiovascular Division, Brigham and Women’s Hospital, 75 Francis Street, Boston, MA 02115. Email: ssolomon@ rics.bwh.harvard.edu 


\section{STRUCTURED ABSTRACT}

Background: Natriuretic peptides are substrates of neprilysin; hence B-type natriuretic peptide (BNP) concentrations rise with neprilysin inhibition. Thus, the clinical validity of measuring BNP in sacubitril/valsartan-treated patients has been questioned, and use of Nterminal pro-BNP (NT-proBNP) has been preferred and recommended.

Objectives: To determine the prognostic performance of BNP measurements before and during treatment with sacubitril/valsartan.

Methods: BNP and NT-proBNP were measured before and after 4-6 weeks, 8-10 weeks, and 9 months of treatment with sacubitril/valsartan in the PARADIGM-HF trial. We assessed the association of levels of these natriuretic peptides with the subsequent risk of cardiovascular death or hospitalization for HF.

Results: Median BNP concentration (before treatment: 202 [Q1-Q3 126-335] ng/L) increased to 235 (Q1-Q3 128-422) ng/L after 8-10 weeks of treatment. A total of 141 (18\%) patients experiencing a doubling and $49(6 \%)$ experienced a tripling of BNP during the first 8-10 weeks of sacubitril/valsartan. In contrast, such striking increases in NT-proBNP following the use of the neprilysin inhibitor were extremely rare. Treatment with sacubitril/valsartan caused a rightward shift in the distribution of BNP when compared with NT-pro-BNP, but both peptides retained their prognostic accuracy (C-statistics of 63-67\% for BNP and Cstatistics of 64-70\% for NT-proBNP) with no difference between the 2 biomarkers. Increases in both BNP and NT-proBNP during 8-10 weeks of sacubitril/valsartan were associated with worse outcomes $(\mathrm{P}=0.003$ and $\mathrm{P}=0.005$, respectively $)$.

Conclusions: Circulating levels of BNP may increase meaningfully early after initiation of sacubitril/valsartan. In comparison, NT-proBNP is less directly influenced by neprilysin inhibition, and thus may lead to less clinical confusion when measured within 8-10 weeks of drug initiation. However, during treatment, either biomarker predicts the risk of major adverse outcomes in patients treated with angiotensin receptor-neprilysin inhibitors.

Clinical Trial Registration: Prospective Comparison of ARNI with ACEI to Determine Impact on Global Mortality and Morbidity in Heart Failure [PARADIGM-HF]; ClinicalTrials.gov Unique identifier: NCT01035255.

Key Words: biomarker; heart failure; natriuretic peptides; prognostication; risk stratification; treatment 


\section{CONDENSED ABSTRACT}

The interpretability of B-type natriuretic peptide (BNP) in sacubitril/valsartan-treated patients is uncertain. We assessed the association between BNP at several time-points (prior to and after 4-6 weeks, 8-10 weeks, and 9 months of treatment) and composite cardiovascular death and heart failure hospitalization in patients treated with sacubitril/valsartan enrolled in the PARADIGM-HF trial. Sacubitril/valsartan led to doubling in BNP after 8-10 weeks in 18\% of patients. Sacubitril/valsartan caused a rightward shift in the distribution of BNP when compared with N-terminal proBNP, but absolute concentrations of both natriuretic peptides during treatment with sacubitril/valsartan were associated with the primary outcome. 


\section{ABBREVIATIONS AND ACRONYMS}

ARNI $=$ angiotensin receptor-neprilysin inhibitor

$\mathrm{BNP}=$ brain natriuretic peptide

$\mathrm{CI}=$ confidence interval

$\mathrm{HR}=$ hazard ratio

$\mathrm{HF}=$ heart failure

$\mathrm{HFrEF}=$ heart failure with reduced ejection fraction

$\mathrm{NP}=$ natriuretic peptide

NT-proBNP $=$ N-terminal pro-B-type natriuretic peptide

PARADIGM-HF = Prospective Comparison of ARNI with ACEI to Determine Impact on Global Mortality and Morbidity in Heart Failure 
1 Natriuretic peptides (NP) are widely employed in routine clinical practice $(1,2)$ for

2 prognostication and risk stratification. C-terminal B-type NP (BNP) has been demonstrated to

3 have similar clinical utility as N-terminal pro-BNP (NT-proBNP) (1,3), and is the only assay

4 available in many hospital systems (4). The angiotensin receptor-neprilysin inhibitor (ARNI)

5 sacubitril/valsartan has been shown to improve cardiovascular outcomes in patients with

6 heart failure with reduced ejection fraction (HFrEF) (5) and is recommended as a

7 replacement for angiotensin-converting enzyme inhibitors (ACEi) or angiotensin II receptor

8 blockers (ARB) $(1,2)$. Neprilysin is a widely expressed enzyme involved in the degradation

9 of several beneficial vasoactive peptides, including NPs. However, while A-type NP (ANP)

10 and C-type NP (CNP) are effectively cleaved by neprilysin, BNP is a relatively poor substrate

11 for neprilysin $(6,7)$. Nevertheless, treatment with sacubitril/valsartan has been associated with

12 an overall increase in BNP, and thus, the clinical utility and interpretability of BNP in

13 sacubitril/valsartan-treated patients has been called into question by clinical practice

14 guidelines, expert consensus statements, and decision pathways (1,8-10). We assessed the

15 relative prognostic value of BNP and NT-proBNP before and during treatment with

16 sacubitril/valsartan in the Prospective Comparison of ARNI with ACEI to Determine Impact

17 on Global Mortality and Morbidity in Heart Failure (PARADIGM-HF) trial.

METHODS

\section{Study design and patient population}

21 PARADIGM-HF was a randomized, double-blind, parallel group, active-controlled trial

22 comparing the long-term efficacy and safety of sacubitril/valsartan compared with enalapril

23 in patients with $\mathrm{HFrEF}$, as previously described $(5,11)$. Patients enrolled had an ejection

24 fraction $\leq 40 \%$ (changed during the trial to $\leq 35 \%$ by amendment), New York Heart 
1 Association (NYHA) class II-IV symptoms, and elevated NPs (if no recent hospitalization for

2 HF: $\mathrm{BNP} \geq 150 \mathrm{ng} / \mathrm{L}$ or N-terminal pro-BNP $\geq 600 \mathrm{ng} / \mathrm{L}$; if hospitalization for HF within 12

3 months: $\mathrm{BNP} \geq 100 \mathrm{ng} / \mathrm{L}$ and NT-proBNP $\geq 400 \mathrm{ng} / \mathrm{L}$ ). Patients were excluded with an

4 estimated glomerular filtration rate (eGFR) $<30 \mathrm{ml} / \mathrm{min} / 1.73 \mathrm{~m}^{2}$, hyperkalemia (potassium

5 concentration $>5.2 \mathrm{mmol} / \mathrm{L}$ at screening or $>5.4 \mathrm{mmol} / \mathrm{L}$ at randomization), hypotension

6 (symptomatic, or a systolic blood pressure $<100 \mathrm{mmHg}$ at screening or $<95 \mathrm{mmHg}$ at

7 randomization), history of angioedema or intolerance to ACEi or ARB. Patients were also

8 required to tolerate $\mathrm{ACEi}$ or $\mathrm{ARB}$ equivalent to enalapril $10 \mathrm{mg}$ daily for $\geq 4$ weeks and be

9 maintained on stable doses of a $\beta$-blocker and mineralocorticoid receptor antagonist (if

10 indicated). Eligible patients entered 4-6 weeks of single-blind enalapril run-in, followed by an

11 additional 4-6 weeks single-blind sacubitril/valsartan run-in. Patients were then randomized

12 in a 1:1 ratio to enalapril $10 \mathrm{mg}$ twice daily or sacubitril/valsartan $200 \mathrm{mg}$ twice daily, if both

13 drugs were tolerated at target dose during the run-in periods. Patients were followed for a

14 mean 2.4 years from randomization and the primary outcome was a composite of death from

15 cardiovascular cause or first hospitalization for HF.

17 BNP and NT-proBNP measurements

18 NP measurements were analyzed in a central laboratory from frozen venous blood samples

19 drawn before run-in $(n=1,656)$, after run-in with both enalapril and sacubitril/valsartan (time

20 of randomization, $n=2,075$ in both study arms), 1 month after randomization ( $n=994$ in the

21 sacubitril/valsartan arm and $\mathrm{n}=1,007$ in the enalapril arm), and 8 months after randomization

22 ( $n=908$ in the sacubitril/valsartan arm and $n=901$ in the enalapril arm). Plasma BNP was

23 measured by the Advia Centaur chemiluminescent immunoassay (Siemens Healthcare

24 Diagnostics, Tarrytown, NY) with a reporting range of 2.7 to $4,590 \mathrm{ng} / \mathrm{L}$. NT-proBNP was 
1 measured by the Roche Elecsys proBNP assay (Roche Diagnostics GmbH, Penzberg,

2 Germany) with a coefficient of variation $<2.5 \%$ at all levels tested between $47 \mathrm{ng} / \mathrm{L}$ and

$3 \quad 34,160 \mathrm{ng} / \mathrm{L}(12)$.

4

\section{$5 \quad$ Statistical analysis}

6 Analyses were performed at 4 time points; before run-in (baseline), at randomization (after 4-

76 weeks of treatment), 1-month post- randomization (after 8-10 weeks of treatment) and 8-

8 months post-randomization (after 9 months of treatment). Patient characteristics are presented

9 by quartiles of BNP concentrations after 8-10 weeks of treatment with sacubitril/valsartan

10 (time point with highest median BNP concentration). Categorical and continuous variables

11 were compared by trend across quartiles using standard parametric or non-parametric methods, as appropriate. BNP and NT-proBNP are presented as median (quartile 1 to quartile 3 [Q1-Q3]). The ratio between NT-proBNP and BNP was calculated by dividing the concentration of NT-proBNP by the concentration in BNP, using the same units. This NP ratio measured early after drug initiation has been hypothesized to identify patients with greater target response to neprilysin inhibition (13).Spearman's rank correlation coefficients $\left(\mathrm{r}_{s}\right)$ were calculated to characterize the correlation between BNP and NT-proBNP at each time-point in sacubitril/valsartan-treated patients. The \% changes in BNP and NT-proBNP during treatment with sacubitril/valsartan were also calculated between pre-run-in (before treatment) and 1 month after randomization (after 8-10 weeks of treatment). Baseline clinical

21 profiles were compared based on BNP trajectory during 8-10 weeks of treatment: increased (greater than $+10 \%)$, stable $( \pm 10 \%)$, or decreased (greater than $-10 \%)$.

24 changes in NPs, and the ratio between NT-proBNP and BNP at each time-point, was 
1 evaluated with respect to the primary outcome. All Cox proportional hazards models included

2 covariates determined a priori based on clinical factors known to influence NP levels and/or

3 clinical outcomes (age, sex, race, body mass index [BMI], history of diabetes mellitus,

4 hypertension, coronary artery disease, follow-up systolic blood pressure, and follow-up eGFR

5 (measured at the contemporaneous time-points as NP concentrations). Model discrimination

6 using BNP and NT-proBNP was estimated and compared using Harrell's C-statistics. For the

7 landmark analysis, we included all patients with available NP measurements who had not

8 experienced the primary outcome prior to this time-point. Only events that occurred after NP

9 sampling were included in the analysis for each time point. For the delta-analysis from before 10 to 8-10 weeks after treatment, only events that occurred after 8-10 weeks were included, and 11 these analyses were also adjusted for baseline NP concentrations before treatment. Kaplan-

12 Meier survival curves were constructed to display time-to-first primary outcomes by quartiles of BNP and NT-proBNP measured at 4-6 weeks and 8-10 weeks of treatment with sacubitril/valsartan. All patients provided written informed consent, and the study was approved by institutional review boards or ethics committees at each participating institution.

16 Statistical analyses were performed using STATA 14.1 (College Station, TX, USA).

\section{RESULTS}

\section{Changes in BNP and NT-proBNP during treatment with sacubitril/valsartan}

Median BNP before both run-in phases and any treatment exposure was 202 (Q1-Q3 126- 
1 assessing only patients in the sacubitril/valsartan arm with available blood samples at all time 2 points $(\mathrm{n}=622)$, as a sensitivity analysis (Online Figure 1).

BNP concentrations at 8-10 weeks of treatment were available in 2,001 (24\%) of all

4 patients in PARADIGM-HF (Table 1). Compared with patients with missing follow-up BNP

5 data, patients with available measurements were older, more likely to be men and white,

6 carried more comorbidities, and had higher blood pressure and lower eGFR (Online Table

7 1). During $8-10$ weeks of treatment with sacubitril/valsartan ( $95 \%$ on target dose), BNP

8 increased by a median of 19\% (Q1 22\% reduction, Q3 75\% increase), with $141(18 \%)$ of

9 patients experiencing doubling and $49(6 \%)$ tripling of BNP during 8-10 weeks of

10 sacubitril/valsartan, while $105(14 \%)$ had stable concentrations ( $\pm 10 \%$ change) (Figure 2$)$. In

11 the same patients, during 8-10 weeks of treatment with sacubitril/valsartan, there was a median reduction in NT-proBNP of 28\% (Q1 52\% reduction, Q3 1\% reduction), with only 18 (2\%) experiencing doubling and $4(0.5 \%)$ experiencing tripling of NT-proBNP during 8-10 weeks of sacubitril/valsartan, while $93(12 \%)$ had stable concentrations $( \pm 10 \%$ change) (Figure 2). In comparison, 8-10 weeks of treatment with enalapril led to a median decrease of $6 \%$ in BNP with $75(10 \%)$ and $31(4 \%)$ experiencing doubling and tripling of BNP levels, respectively. Similarly, a median decrease of 5\% in NT-proBNP was observed during enalapril treatment with $53(7 \%)$ and $18(2 \%)$ experiencing double and tripling of NTproBNP levels, respectively. Overall, treatment with sacubitril/valsartan shifted the distribution of BNP concentrations rightward compared with that of NT-proBNP. Patients increasing (more than $+10 \%$ ) in BNP during 8-10 weeks of treatment with sacubitril/valsartan were older, more likely to be male, had higher prevalence of previous myocardial infarction, and ischemic cardiomyopathy, and had lower eGFR and BNP concentrations before treatment compared with patients decreasing (more than -10\%) in BNP 
1 (Online Table 2). Patients experiencing a doubling or more in BNP during 8-10 weeks of

2 treatment with sacubitril/valsartan were older $(69 \pm 9$ years vs. $67 \pm 11$ years, $p=0.009)$ with

3 lower baseline, pre-treatment BNP (164 [Q1-Q3 106-345] ng/L vs. 212 [Q1-Q3 135-333]

$4 \mathrm{ng} / \mathrm{L}, \mathrm{p}=0.02$ ) compared with patients that decreased or increased $<100 \%$ in BNP.

5

6 Prognostic value of BNP and NT-proBNP before and during treatment with

7 sacubitril/valsartan

8 BNP and NT-proBNP had comparable prognostic performance in the total cohort at screening $9(\mathrm{n}=8,348 ; \mathrm{C}$-statistics $64.0 \%$ and $63.6 \%$, respectively, $\mathrm{p}=0.37)$. Concentrations of $\mathrm{BNP}$ and 10 NT-proBNP correlated strongly before treatment with sacubitril/valsartan $\left(\mathrm{r}_{s}=0.77\right)$, and at each of the time-points after treatment initiation $\left(\mathrm{r}_{s}=0.80\right.$ at 4-6 weeks of treatment, $\mathrm{r}_{s}=0.75$ at 8-10 weeks of treatment, and $r_{s}=0.78$ at 9 months of treatment). Log-transformed concentrations of BNP and NT-proBNP were strongly associated with the primary endpoint at each time-point, independent of key demographic and clinical factors $(\mathrm{P}<0.001$ for all time-points; Table 2). Survival curves by quartiles of BNP and NT-proBNP after 4-6 weeks of treatment are displayed in Online Figure 2 and after 8-10 weeks of treatment in Online Figure 3. C-statistics for the primary endpoint ranged from $63 \%$ to $67 \%$ for BNP and from $64 \%$ to $70 \%$ for NT-proBNP, and there were no significant differences between the 2 biomarkers in predicting outcome at any of the time-points before and during treatment with sacubitril/valsartan ( $\mathrm{P}>0.05$ for all time-points; Table 2). The association between quartiles of BNP and NT-proBNP and subsequent primary outcomes is presented in Figure 3 (Central Illustration). during 8-10 weeks of treatment with sacubitril/valsartan were associated with the primary 
1 outcome, even after accounting for demographics, comorbidities, blood pressure, eGFR, and

2 baseline NP levels prior to treatment (Figure 2).

\section{Ratio between NT-proBNP and BNP}

5 The median ratio between NT-proBNP and BNP was 6.3 (Q1-Q3 4.5-8.8) before treatment 6 and was $3.8(2.8-5.5)$ at 4-6 weeks, $3.8(2.7-5.3)$ at $8-10$ weeks, and $3.9(2.7-5.7)$ at 9 months

7 of treatment with sacubitril valsartan. In contrast, the median ratio of NT-proBNP and BNP

8 did not change with 8-10 weeks (6.5 [4.7-9.2]) and 9 months (6.4 [4.7-9.2]) of treatment with

9 enalapril. The NT-proBNP:BNP ratio at each time-point was not independently associated

10 with the primary outcome in sacubitril/valsartan-treated patients $(\mathrm{P}>0.10$ for each of the time11 points; Online Table 3).

\section{DISCUSSION}

14 Sacubitril/valsartan led to meaningful increases in BNP concentrations in many patients with

15 peak levels detected at 8-10 weeks during treatment. Sacubitril/valsartan shifts the

16 distribution of BNP concentrations rightward early after treatment initiation. In contrast, NT-

17 proBNP is less directly influenced by sacubitril/valsartan, and its measurement may be preferred within 8-10 weeks of drug initiation. However, despite its initial rise, on-treatment BNP concentrations remained robustly and independently associated with adverse cardiovascular outcomes, with comparable discrimination of risk to that of on-treatment NTproBNP concentrations. Greater relative increases in BNP and NT-proBNP during treatment with sacubitril/valsartan were independently associated with higher risk of the primary outcome. 


\section{$1 \quad$ Natriuretic peptide biology}

2 NPs are key regulators of volume and blood pressure homeostasis, and are upregulated as a

3 compensatory mechanism to cardiomyocyte stretch (14). The beneficial effects of NPs occur

4 through a complex signaling system that involves upregulation of intracellular cyclic

5 guanosine monophosphate (cGMP), which induces vasodilation and excretion of water and

6 sodium in the kidneys. Neprilysin is the key enzyme responsible for the breakdown of these

7 peptides. Neprilysin cleaves ANP and CNP efficiently, while BNP is a poorer substrate and

8 the inactive fragment NT-proBNP is not affected by neprilysin $(6,7,15)$. In PARADIGM-HF,

9 combined neprilysin and renin-angiotensin-aldosterone-system inhibition with

10 sacubitril/valsartan was associated with lower cardiovascular mortality and hospitalization for

11 HF compared with renin-angiotensin-aldosterone-system inhibition alone (5). The marked

12 increase in urinary cGMP observed in patients treated with sacubitril/valsartan suggests that

13 enhanced intracellular effects of NPs may be an important pharmacodynamic mechanism

14 related to the drug (16).

16 Measurement of NPs in sacubitril/valsartan-treated patients in clinical practice

17 On average, treatment with sacubitril/valsartan has been recognized to be associated with an initial increase in BNP and decrease in NT-proBNP (5). As such, current clinical guidelines (1) and expert consensus statements (8) call into question the utility of on-treatment BNP testing. For instance, the 2017 American College of Cardiology Expert Consensus Decision

21 Pathway (8) states that "BNP concentrations will increase (while NT-proBNP will most often fall) with ARNI therapy, and thus it may be more prudent to check only NT-proBNP in patients on ARNI." 
In the present study, we provide additional data demonstrating that increases in BNP

2 are modest (on average, $\sim 20 \%$ increases) in most patients, but more substantial in some

3 ( 20\% of patients experience doubling of baseline levels after ARNI initiation). BNP

4 increases occur early after treatment initiation (8-10 weeks peak effect). As such, until BNP

5 reaches a "steady state" during the maintenance phase of ARNI treatment, measurement of

6 NT-proBNP, which is much less subject to direct drug effects, may introduce less clinical

7 confusion and is preferred.

However, our data suggest that both biomarkers convey similar prognostic information

9 given the relatively uniform rightward shift in BNP concentrations by sacubitril/valsartan.

10 BNP carried a consistent association with cardiovascular events before and during treatment

11 with sacubitril/valsartan. Baseline (pre-treatment) levels of NPs, potentially reflecting background risk, are important determinants of subsequent measurements, even in the presence of a neprilysin inhibitor and when either BNP or NT-proBNP assays are used. As such, the prognostic performance of on-treatment BNP levels was comparable to NT-proBNP at every time-point and there was a consistent and strong correlation between NT-proBNP and BNP both before and during treatment with sacubitril/valsartan.

Taken together, although BNP concentrations are increased with initiation of sacubitril/valsartan, on-treatment measurement remains reliable in predicting risk. Natriuretic peptides may be strongly influenced by comorbid diseases and adiposity, genetic determinants and race/ethnicity, physiological states (such as pregnancy), and severity of HF.

21 We tested the hypothesis of whether pharmacological modification of the distribution of BNP concentrations would influence its prognostic value. Similar to certain populations (such as atrial fibrillation) where the entire biomarker distribution is shifted rightward, but remain 
1 interpretable and meaningful (17), absolute and relative changes in NPs remain

2 prognostically important in ARNI-treated patients, regardless of assay. are consistently associated with adverse cardiovascular risk. Given the modest increases BNP concentrations after treatment initiation (especially relative to baseline values) in most

6 patients and known long-term cardiovascular benefits of sacubitril/valsartan, the prognostic

7 relevance of more substantial BNP increases likely reflects disease progression rather than

8 blunted NP degradation by sacubitril/valsartan alone. Early improvement or worsening in HF

9 status influences BNP changes and may overshadow the relatively modest direct ARNI-

10 related effects on NP clearance. Consistently, in the PIONEER-HF (comParIson Of sacubitril/valsartaN versus Enalapril on Effect on nt-pRo-bnp in patients stabilized from an acute Heart Failure episode) trial (18), both NT-proBNP and BNP fell over 4-8 weeks after in-hospital initiation of either sacubitril/valsartan or enalapril, likely driven by net postdischarge improvement in congestion status and compensation of HF. Given the competing contributions of disease activity and blunted NP degradation, we were unable to identify an expected or acceptable rise in BNP after sacubitril/valsartan initiation. However, given the potential effects of disease activity on serial BNP concentrations in ARNI-treated patients, all BNP increases should not be dismissed as being related to drug effects alone. Although BNP predicts adverse cardiovascular risk in sacubitril/valsartan-treated patients, it should not be used in clinical decision-making regarding treatment continuation.

21 Specifically, early modest rises in BNP which may be expected with starting the drug should not be a reason that sacubitril/valsartan is dose-reduced, interrupted, or discontinued in clinical practice. In most sacubitril/valsartan-treated patients, BNP concentrations are expected to reach steady-state or decline after several months of maintenance dosing. 
1 Although NT-proBNP-to-BNP ratio after initiation of sacubitril/valsartan has previously been

2 suggested as clinically meaningful (13), we did not observe an association between this NP

3 ratio and clinical outcomes at any time-point during treatment with sacubitril/valsartan.

4

\section{Study limitations}

6 The study is subject to several limitations. First, the analyses were limited to the subset of

7 patients with available NP concentrations that in general carried more cardiovascular risk

8 factors compared with the original PARADIGM-HF trial. Second, we relied on pre-treatment

9 measurements of NPs from prior to run-in of both enalapril and sacubitril/valsartan

$10(n=1,656)$, because the number of available blood samples between run-in with enalapril and sacubitril/valsartan was limited $(n=903)$. However, the run-in with enalapril did not change BNP concentrations (before: median BNP 198 [Q1-Q3 123-340] ng/L vs. after: 198 [123340]. Third, we only used one assay to analyze BNP and NT-proBNP. Given the substantial assay-specific, glycosylation-dependent $(19,20)$, cross-reactivity of the precursor pro-BNP with commercial BNP and NT-proBNP assays, different assays could potentially detect different epitopes on the peptide $(15,21)$. Although certain NP assays have been shown to be stable when stored under freezing conditions (22), freeze-thaw cycles required for processing and measurement by the central laboratory may have influenced concentrations. Mechanistic studies, such as PROVE-HF (Prospective Study of Biomarkers, Symptom Improvement, and Ventricular Remodeling During Sacubitril/Valsartan Therapy for Heart Failure;

ClinicalTrials.gov identifier: NCT02887183), are underway and are anticipated to provide comprehensive understanding of the effects of neprilysin inhibition on a broad range of biomarkers (23). Finally, early BNP trajectories may differ in patients by baseline neprilysin levels, which were not measured in this study. 


\section{CONCLUSIONS}

2 Sacubitril/valsartan increases BNP early after treatment initiation to a modest extent ( 20\%)

3 in most, but more substantially in some treated patients. As NT-proBNP is the least

4 vulnerable to degradation by neprilysin and thus relatively less subject to direct ARNI effects,

5 its measurement (if available) is preferred to limit clinical confusion during initial drug

6 initiation, consistent with current recommendations (8). However, in hospital systems where

$7 \quad$ BNP is the only NP-assay available, its measurement during treatment with

8 sacubitril/valsartan reliably reflects clinical prognosis with comparable performance to NT-

9 proBNP. Importantly, early increases after drug initiation in either NP level, especially to a

10 greater magnitude, should not be ascribed to drug effects alone, and identify patients at

11 heightened risk for clinical events. As such, BNP or NT-proBNP can continue to be used

12 based on local laboratory availability in monitoring risk by clinicians (as indicated) in

13 sacubitril/valsartan-treated patients. Given short-term variability in BNP responses to

14 treatment with sacubitril/valsartan, this marker should not however be used to determine

15 treatment adherence or degree of treatment response, or lack thereof, in individual patients.

16 Although BNP is right-shifted during treatment with sacubitril/valsartan which may cause

17 confusion in the initial phases, it remains an important and clinically valid biomarker that

18 carries independent prognostic value in patients treated with sacubitril/valsartan that is

19 similar to that of NT-proBNP. 


\section{CLINICAL PERSPECTIVES}

Competency in Medical Knowledge: Sacubitril/valsartan causes an early, but modest rise in BNP levels in most treated patients.

Competency in Patient Care and Procedural Skills: BNP remains a reliable marker of prognosis and can continue to be used in monitoring risk (as indicated) in sacubitril/valsartantreated patients.

Translational Outlook: Future mechanistic studies, such as PROVE-HF (Prospective Study of Biomarkers, Symptom Improvement, and Ventricular Remodeling During Sacubitril/Valsartan Therapy for Heart Failure), will define the temporal profile of effects of neprilysin inhibition on the broad spectrum of NPs and assays used to detect them. 


\section{REFERENCES}

1. Yancy CW, Jessup M, Bozkurt B et al. 2017 ACC/AHA/HFSA Focused Update of the 2013 ACCF/AHA Guideline for the Management of Heart Failure: A Report of the American College of Cardiology/American Heart Association Task Force on Clinical Practice Guidelines and the Heart Failure Society of America. Circulation 2017.

2. Ponikowski P, Voors AA, Anker SD et al. 2016 ESC Guidelines for the diagnosis and treatment of acute and chronic heart failure: The Task Force for the diagnosis and treatment of acute and chronic heart failure of the European Society of Cardiology (ESC)Developed with the special contribution of the Heart Failure Association (HFA) of the ESC. Eur Heart J 2016;37:2129-200.

3. Clerico A, Fontana M, Zyw L, Passino C, Emdin M. Comparison of the diagnostic accuracy of brain natriuretic peptide (BNP) and the $\mathrm{N}$-terminal part of the propeptide of BNP immunoassays in chronic and acute heart failure: a systematic review. Clin Chem 2007;53:813-22.

4. Hammerer-Lercher A, Collinson P, van Dieijen-Visser Marja P et al. Do laboratories follow heart failure recommendations and guidelines and did we improve? The CARdiac MArker Guideline Uptake in Europe (CARMAGUE). Clin Chem Lab Med 2013:1301.

5. McMurray JJ, Packer M, Desai AS et al. Angiotensin-neprilysin inhibition versus enalapril in heart failure. N Engl J Med 2014;371:993-1004.

6. Pankow K, Schwiebs A, Becker M, Siems WE, Krause G, Walther T. Structural substrate conditions required for neutral endopeptidase-mediated natriuretic Peptide degradation. J Mol Biol 2009;393:496-503.

7. Walther T, Stepan H, Pankow K, Becker M, Schultheiss HP, Siems WE. Biochemical analysis of neutral endopeptidase activity reveals independent catabolism of atrial and brain natriuretic peptide. Biol Chem 2004;385:179-84.

8. Yancy CW, Januzzi JL, Jr., Allen LA et al. 2017 ACC Expert Consensus Decision Pathway for Optimization of Heart Failure Treatment: Answers to 10 Pivotal Issues About Heart Failure With Reduced Ejection Fraction: A Report of the American College of Cardiology Task Force on Expert Consensus Decision Pathways. J Am Coll Cardiol 2018;71:201-230. 
9. Lippi G, Sanchis-Gomar F. Monitoring B-type natriuretic peptide in patients undergoing therapy with neprilysin inhibitors. An emerging challenge? Int J Cardiol 2016;219:111-4.

10. Ibrahim NE, Januzzi J. Monitoring Biomarkers in Patients Receiving Neprilysin Inhibitors. Curr Emerg Hosp Med Rep 2018;6:8-16.

11. McMurray JJ, Packer M, Desai AS et al. Dual angiotensin receptor and neprilysin inhibition as an alternative to angiotensin-converting enzyme inhibition in patients with chronic systolic heart failure: rationale for and design of the Prospective comparison of ARNI with ACEI to Determine Impact on Global Mortality and morbidity in Heart Failure trial (PARADIGM-HF). Eur J Heart Fail 2013;15:1062-73.

12. Zile MR, Claggett BL, Prescott MF et al. Prognostic Implications of Changes in NTerminal Pro-B-Type Natriuretic Peptide in Patients With Heart Failure. J Am Coll Cardiol 2016;68:2425-2436.

13. Mair J, Lindahl B, Giannitsis E et al. Will sacubitril-valsartan diminish the clinical utility of B-type natriuretic peptide testing in acute cardiac care? Eur Heart J Acute Cardiovasc Care 2017;6:321-328.

14. Volpe M, Rubattu S, Burnett JJ. Natriuretic peptides in cardiovascular diseases: current use and perspectives. Eur Heart J 2014;35:419-425.

15. Semenov AG, Katrukha AG. Different Susceptibility of B-Type Natriuretic Peptide (BNP) and BNP Precursor (proBNP) to Cleavage by Neprilysin: The N-Terminal Part Does Matter. Clin Chem 2016;62:617-622.

16. Packer M, McMurray JJ, Desai AS et al. Angiotensin receptor neprilysin inhibition compared with enalapril on the risk of clinical progression in surviving patients with heart failure. Circulation 2015;131:54-61.

17. Myhre PL, Vaduganathan M, Claggett BL et al. Association of Natriuretic Peptides With Cardiovascular Prognosis in Heart Failure With Preserved Ejection Fraction: Secondary Analysis of the TOPCAT Randomized Clinical Trial. JAMA Cardiol 2018.

18. Velazquez EJ, Morrow DA, DeVore AD et al. Angiotensin-Neprilysin Inhibition in Acute Decompensated Heart Failure. N Engl J Med 2018 (in press).

19. Seferian KR, Tamm NN, Semenov AG et al. Immunodetection of glycosylated NTproBNP circulating in human blood. Clin Chem 2008;54:866-73. 
20. Mair J. Clinical significance of pro-B-type natriuretic peptide glycosylation and processing. Clin Chem 2009;55:394-7.

21. Luckenbill KN, Christenson RH, Jaffe AS et al. Cross-reactivity of BNP, NTproBNP, and proBNP in commercial BNP and NT-proBNP assays: preliminary observations from the IFCC Committee for Standardization of Markers of Cardiac Damage. Clin Chem 2008;54:619-21.

22. Nowatzke WL, Cole TG. Stability of N-terminal pro-brain natriuretic peptide after storage frozen for one year and after multiple freeze-thaw cycles. Clin Chem 2003;49:1560-2.

23. Januzzi JL, Butler J, Fombu E et al. Rationale and methods of the Prospective Study of Biomarkers, Symptom Improvement, and Ventricular Remodeling During Sacubitril/Valsartan Therapy for Heart Failure (PROVE-HF). Am Heart J 2018;199:130-136. 


\section{FIGURE LEGENDS}

\section{Figure 1. Natriuretic Peptide Trajectories in the PARADIGM-HF Trial.}

Median concentration of B-type natriuretic peptide (BNP) and N-terminal pro-B-type natriuretic peptide (NT-proBNP) before (V2) and during (V5, V7, V10) treatment with sacubitril/valsartan and enalapril.

Figure 2. Distribution of Natriuretic Peptide Responses after 8-10 Week Treatment with Sacubitril/Valsartan.

The gold histogram represents changes in B-type natriuretic peptide (BNP) and N-terminal pro-BNP (NT-proBNP) during 8-10 weeks of treatment with sacubitril/valsartan, presented as $\%$ change from pre-run-in to 1 month after randomization. The histogram corresponds to the secondary (right-sided) Y-axis.

The solid black line represents an estimation by Poisson regression of the association between change in natriuretic peptides and the primary outcome after adjusting for age, sex, race, body mass index, diabetes mellitus, hypertension, coronary artery disease, systolic blood pressure, estimated glomerular filtration rate, and pre-run-in natriuretic peptide concentrations. Dotted lines represent the $95 \%$ confidence intervals. Incidence rates are displayed on the primary (left-sided) Y-axis.

Figure 3 (Central Illustration). Association Between Natriuretic Peptide Concentrations and Subsequent Cardiovascular Events Before and During Treatment with Sacubitril/Valsartan. 
Association between log-transformed B-type natriuretic peptide (BNP) and N-terminal proB-type natriuretic peptide (NT-proBNP) and the primary outcome before (V2) and during (V5, V7 and V10) treatment with sacubitril/valsartan after adjusting for age, sex, race, body mass index, diabetes mellitus, hypertension, coronary artery disease, and systolic blood pressure at the same visit, estimated glomerular filtration rate at the same visit. Spearman's rank correlation coefficients $\left(\mathrm{r}_{s}\right)$ were calculated to characterize the correlation between BNP and NT-proBNP at each time-point. Abbreviations: $\mathrm{CI}=$ confidence interval; $\mathrm{HR}=$ hazard ratio. 
Table 1 Patient characteristics according to quartiles of BNP levels at 8-10 weeks of treatment with sacubitril/valsartan (sacubitril/valsartan arm only)

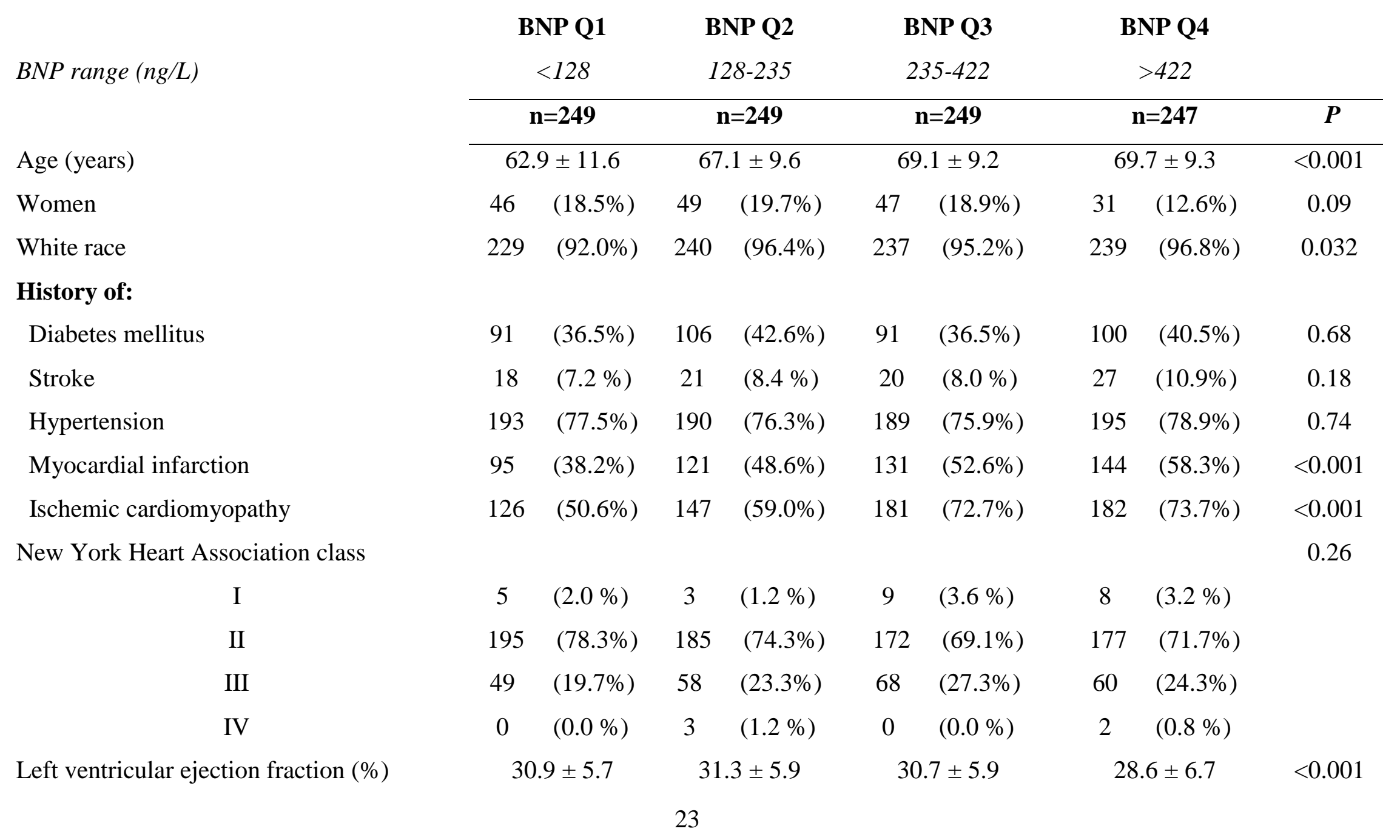




\section{Background therapies}

\begin{tabular}{|c|c|c|c|c|c|c|c|c|c|}
\hline Angiotensin-converting enzyme inhibitor & 209 & $(83.9 \%)$ & 204 & $(81.9 \%)$ & 206 & $(82.7 \%)$ & 197 & $(79.8 \%)$ & 0.28 \\
\hline Angiotensin II receptor blocker & 42 & $(16.9 \%)$ & 46 & $(18.5 \%)$ & 44 & $(17.7 \%)$ & 52 & $(21.1 \%)$ & 0.29 \\
\hline Mineralocorticoid receptor antagonist & 118 & $(47.4 \%)$ & 94 & $(37.8 \%)$ & 104 & $(41.8 \%)$ & 103 & $(41.7 \%)$ & 0.35 \\
\hline Diuretic & 198 & $(79.5 \%)$ & 197 & $(79.1 \%)$ & 196 & $(78.7 \%)$ & 215 & $(87.0 \%)$ & 0.05 \\
\hline Beta-blocker & 240 & $(96.4 \%)$ & 237 & $(95.2 \%)$ & 233 & $(93.6 \%)$ & 240 & $(97.2 \%)$ & 0.90 \\
\hline Cardiac resynchronization therapy & 20 & $(8.0 \%)$ & 24 & $(9.6 \%)$ & 32 & $(12.9 \%)$ & 28 & $(11.3 \%)$ & 0.13 \\
\hline Implantable cardioverter-defibrillator & 68 & $(27.3 \%)$ & 60 & $(24.1 \%)$ & 72 & $(28.9 \%)$ & 79 & $(32.0 \%)$ & 0.14 \\
\hline Measurements at Visit 7 & & & & & & & & & \\
\hline Systolic blood pressure (mmHg) & \multicolumn{2}{|c|}{$123.1 \pm 15.6$} & \multicolumn{2}{|c|}{$123.5 \pm 17.5$} & \multicolumn{2}{|c|}{$123.0 \pm 17.7$} & \multicolumn{2}{|c|}{$123.7 \pm 18.2$} & 0.75 \\
\hline \multicolumn{10}{|l|}{ Estimated glomerular filtration rate } \\
\hline$\left(\mathrm{mL} / \mathrm{min} / \mathrm{m}^{2}\right)$ & \multicolumn{2}{|c|}{$66.4 \pm 19.0$} & \multicolumn{2}{|c|}{$65.5 \pm 17.3$} & \multicolumn{2}{|c|}{$63.3 \pm 15.6$} & \multicolumn{2}{|c|}{$58.9 \pm 17.3$} & $<0.001$ \\
\hline Potassium (mmol/L) & \multicolumn{2}{|c|}{$4.6 \pm 0.5$} & \multicolumn{2}{|c|}{$4.5 \pm 0.4$} & \multicolumn{2}{|c|}{$4.5 \pm 0.4$} & \multicolumn{2}{|c|}{$4.4 \pm 0.5$} & 0.006 \\
\hline NT-proBNP (ng/L) & \multicolumn{2}{|c|}{$357[221,580]$} & \multicolumn{2}{|c|}{$682[481,969]$} & \multicolumn{2}{|c|}{$1162[808,1510]$} & \multicolumn{2}{|c|}{$2346[1551,4011]$} & $<0.001$ \\
\hline
\end{tabular}

Abbreviations: $\mathrm{BNP}=\mathrm{B}$-type natriuretic peptide; NT-proBNP $=\mathrm{N}$-terminal pro-B-type natriuretic peptide. 
Table 2 Association between log-transformed concentrations of natriuretic peptides and the primary outcome before and during treatment with sacubitril/valsartan

\begin{tabular}{|c|c|c|c|c|c|c|c|c|}
\hline & \multirow{2}{*}{$\begin{array}{c}\text { Events, } \\
\text { incidence rates }(95 \% \\
\text { CI) per } 100 \text { p.y. }\end{array}$} & \multicolumn{3}{|c|}{ BNP (log-transformed) } & \multicolumn{3}{|c|}{ NT-proBNP (log-transformed) } & \multirow{2}{*}{$\begin{array}{c}P \text { for } \\
\text { difference in } \\
\text { C-statistics }\end{array}$} \\
\hline & & $\begin{array}{c}\text { Unadjusted HR } \\
(95 \% \mathrm{CI})\end{array}$ & $\begin{array}{l}\text { C-statistics } \\
(95 \% \mathrm{CI})\end{array}$ & $\begin{array}{c}\text { Adjusted HR } \\
(95 \% \mathrm{CI})^{*}\end{array}$ & $\begin{array}{c}\text { Unadjusted HR } \\
(95 \% \mathrm{CI})\end{array}$ & $\begin{array}{c}\text { C-statistics } \\
(95 \% \mathrm{CI})\end{array}$ & $\begin{array}{l}\text { Adjusted HR } \\
(95 \% \mathrm{CI})^{*}\end{array}$ & \\
\hline $\begin{array}{c}\text { V2 }(\mathbf{n = 1 , 6 5 6 )} \\
\text { Before treatment with } \\
\text { sacubitril/valsartan }\end{array}$ & $\begin{array}{c}n=370, \\
9.41(8.50-10.42)\end{array}$ & $\begin{array}{c}1.64 \\
(1.46-1.85)\end{array}$ & $\begin{array}{c}63 \% \\
(60-66 \%)\end{array}$ & $\begin{array}{c}1.75 \\
(1.49-2.05)\end{array}$ & $\begin{array}{c}1.68 \\
(1.50-1.88)\end{array}$ & $\begin{array}{c}64 \% \\
(61-67 \%)\end{array}$ & $\begin{array}{c}1.89 \\
(1.62-2.21)\end{array}$ & 0.42 \\
\hline $\begin{array}{c}\text { V5 }(\mathbf{n = 2 , 0 7 5 )} \\
\text { After 4-6 weeks with } \\
\text { sacubitril/valsartan }\end{array}$ & $\begin{array}{c}\mathrm{n}=464 \\
10.02(9.15-10.98)\end{array}$ & $\begin{array}{c}1.58 \\
(1.45-1.73)\end{array}$ & $\begin{array}{c}65 \% \\
(62-68 \%)\end{array}$ & $\begin{array}{c}1.60 \\
(1.45-1.77)\end{array}$ & $\begin{array}{c}1.74 \\
(1.58-1.92)\end{array}$ & $\begin{array}{c}65 \% \\
(63-68 \%)\end{array}$ & $\begin{array}{c}1.80 \\
(1.62-2.01)\end{array}$ & 0.58 \\
\hline $\begin{array}{c}\text { V7 }(\mathbf{n}=994) \\
\text { After } 8-10 \text { weeks with } \\
\text { sacubitril/valsartan }\end{array}$ & $\begin{array}{c}\mathrm{n}=194 \\
8.94(7.76-10.29)\end{array}$ & $\begin{array}{c}1.80 \\
(1.55-2.09)\end{array}$ & $\begin{array}{c}67 \% \\
(62-71 \%)\end{array}$ & $\begin{array}{c}1.71 \\
(1.45-2.01)\end{array}$ & $\begin{array}{c}2.09 \\
(1.80-2.45)\end{array}$ & $\begin{array}{c}68 \% \\
(64-72 \%)\end{array}$ & $\begin{array}{c}2.01 \\
(1.69-2.39)\end{array}$ & 0.29 \\
\hline $\begin{array}{l}\text { V10 }(\mathbf{n = 9 0 8}) \\
\text { After } 9 \text { months with } \\
\text { sacubitril/valsartan }\end{array}$ & $\begin{array}{c}\mathrm{n}=124, \\
7.80(6.54-9.30)\end{array}$ & $\begin{array}{c}1.77 \\
(1.47-2.13)\end{array}$ & $\begin{array}{c}67 \% \\
(62-72 \%)\end{array}$ & $\begin{array}{c}1.73 \\
(1.41-2.12)\end{array}$ & $\begin{array}{c}2.10 \\
(1.74-2.53)\end{array}$ & $\begin{array}{c}70 \% \\
(66-75 \%)\end{array}$ & $\begin{array}{c}2.12 \\
(1.71-2.62)\end{array}$ & 0.06 \\
\hline
\end{tabular}

*Adjusted for age, sex, race, body mass index, diabetes mellitus, hypertension, coronary artery disease, and systolic blood pressure at the same

visit, and estimated glomerular filtration rate at the same visit, Abbreviations: $\mathrm{CI}=$ confidence interval; $\mathrm{HR}=$ hazard ratio 